RELATO DE CASO

\title{
Aneurisma verdadeiro da artéria radial: relato de caso
}

\author{
True aneurysm of the radial artery: a case report \\ Claudio Nacif Feres', Marcos Augusto de Araújo Ferreira'
}

\begin{abstract}
Resumo
Aneurismas da artéria radial são raros e geralmente estão relacionados com traumas penetrantes. Um caso de aneurisma verdadeiro do segmento distal da artéria radial foi descrito com ênfase na sua apresentação extremamente rara. Aneurismectomia com ligadura proximal e distal dos cotos arteriais foi realizada com sucesso.
\end{abstract}

Palavras-chave: Aneurisma; artéria radial, aneurisma.

\begin{abstract}
Aneurysms of the radial artery are rare and often associated with penetrating trauma. A true case of radial artery distal segment aneurysm was described with emphasis on its extremely rare clinical presentation. Aneurysmectomy with proximal and distal ligatures of the arterial stumps was performed with success.
\end{abstract}

Keywords: Aneurysm; radial artery, aneurysm.

\section{Introdução}

Aneurismas são definidos como uma dilatação localizada e permanente de uma artéria com um aumento de mais de $50 \%$ em seu diâmetro, comparado com as medidas consideradas normais. Os aneurismas são geralmente classificados pela localização, causa e aparência. Em relação à causa, são classificados em traumáticos e não-traumáticos. Causas não-traumáticas de aneurismas arteriais periféricos incluem infecções fúngicas, aterosclerose, causas inflamatórias e idiopáticas ${ }^{1-4}$. Os aneurismas verdadeiros das artérias dos membros superiores são relativamente raros $^{1}$.

No presente estudo, relata-se um caso de aneurisma verdadeiro de artéria radial, sua apresentação clínica e exames complementares diagnósticos.

\section{Relato de caso}

Paciente do sexo feminino, 47 anos, branca, apresentava há 6 meses um tumor no punho direito, de aparecimento espontâneo, com aumento progressivo, sem dor ou outra manifestação clínica. A paciente não apresentava história de traumas, infecções ou punções na região. Ao exame físico, evidenciou-se tumor pulsátil no trajeto da artéria radial direita, que media aproximadamente $1,2 \mathrm{~cm}$ de diâmetro. Foram solicitados ecodoppler da artéria radial direita, arteriografia do membro superior direito e ressonância magnética de crânio.

O ecodoppler demonstrou a presença de dilatação aneurismática da artéria radial direita com $1,1 \times 1,0 \mathrm{~cm}$ no seu maior diâmetro, com imagens ecogênicas de 0,56 x $0,38 \mathrm{~cm}$ na parede posterior e $0,74 \times 0,29 \mathrm{~cm}$ na parede anterior, sugestiva de trombos. A arteriografia evidenciou imagem compatível com aneurisma trombosado no segmento distal da artéria radial direita (Figura 1). A ressonância magnética nuclear de crânio não apresentou anormalidades. A suspeita inicial de aneurisma da artéria radial direita foi confirmada e, diante da história clínica supracitada, definiu-se como hipótese diagnóstica aneurisma verdadeiro de artéria radial direita.

A paciente foi submetida a tratamento cirúrgico, sendo realizada uma aneurismectomia com ligadura dos cotos proximal e distal da artéria radial direita, sem intercorrências. O estudo anatomopatológico da peça cirúrgica foi compatível com aneurisma arterial verdadeiro com trombose mural da artéria radial direita.

A paciente vinha sendo seguida ambulatorialmente há três anos, com resultado satisfatório, sem sinais de outros aneurismas. 

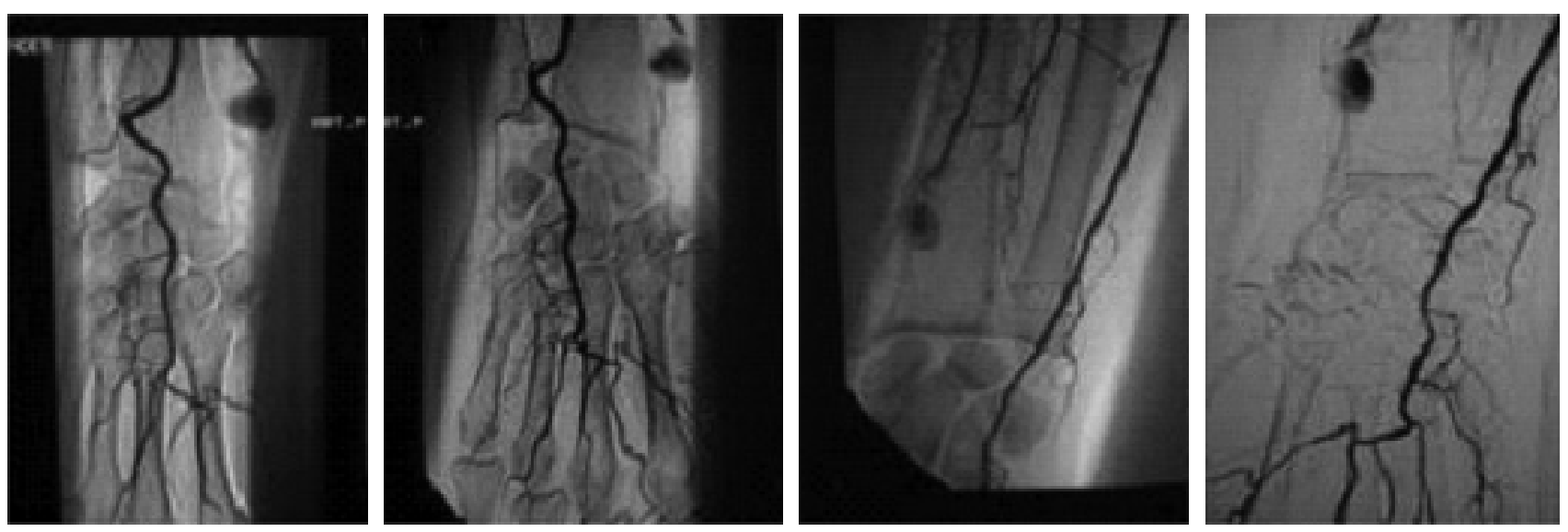

Figura 1 - Arteriografia mostrando aneurisma da artéria radial com trombo em seu interior.

\section{Discussão}

Aneurisma da artéria radial é raro e, usualmente, secundário a traumas penetrantes. A artéria radial é comumente afetada no seu segmento distal, no qual se localiza mais superficialmente. O aneurisma verdadeiro está frequentemente associado a traumatismos não-penetrantes, como contusões 5 . O aneurisma falso decorre de trauma penetrante da parede arterial com formação de hematoma. Exames histológicos mostram a presença de um trombo organizado e uma parede fibrosa que não contém os elementos normais de uma parede $\operatorname{arterial}^{1}$. Não há evidências de fibras musculares ou elásticas na sua parede arterial, comparado com os aneurismas verdadeiros.

Os raros casos de aneurisma da artéria radial descritos na literatura estão relacionados com punção e/ou cateterismo arterial e associados a doenças como neurofibromatose, anemia severa e doença de Buerger ${ }^{6-8}$.

Neste artigo, foi relatado um caso de aneurisma idiopático da artéria radial, que se caracterizava quanto a sua apresentação clínica por ser assintomático e sem história prévia de trauma penetrante e outras doenças associadas ${ }^{910}$. A importância clínica do caso se deve à sua raridade ${ }^{11}$. Após confirmação diagnóstica com exames de ecodoppler e arteriografia, a paciente foi submetida a tratamento cirúrgico, optando-se por aneurismectomia sem reconstrução arterial.

\section{Referências}

1. Ho PK, Weiland AJ, McClinton MA, Wilgis EF. Aneurysms of the upper extremity. J Hand Surg Am. 1987;12:39-46.

2. Berrettoni BA, Seitz WH Jr. Mycotic aneurysm in a digital artery: case report and literature review. J Hand Surg Am. 1990;15:305-8.

3. Strauch B, Melone C, McClain SA, Lee BT. True aneurysms of the digital artery: case report. J Hand Surg Am. 2004;29:54-8.
4. Leitner DW, Ross JS, Neary JR. Granulomatous radial arteritis with bilateral, nontraumatic, true arterial aneurysms within the anatomic snuffbox. J Hand Surg Am. 1985;10:131-5.

5. Behar JM, Winston JS, Knowles J, Myint F. Radial artery aneurysm resulting from repetitive occupational injury: Tailor's thumb. Eur J Vasc Endovasc Surg. 2007;34:299-301.

6. Singh S, Riaz M, Wilmshurst AD, Small JO. Radial artery aneurysm in a case of neurofibromatosis. Br J Plast Surg. 1998;51:564-5.

7. Coppola G, Amann-Vesti BR, Koppensteiner R. Aneurysms of radial arteries and severe anemia. Vasa. 2003;32:178.

8. Giler S, Zelikovski A, Goren G, Urca I. Aneurysm of the radial artery in a patient with Buerger's disease. Vasa. 1979;8:147-9.

9. Santos ACB, Oliveira FM, Oliveira JG, et al. Aneurisma idiopático da artéria radial na região da tabaqueira anatômica: relato de caso. I Vasc Bras. 2008;7:380-3.

10. Walton NP, Choudhary F. Idiopathic radial artery aneurysm in the anatomical snuff box. Acta Orthop Belg. 2002;68:292-4.

11. Khatrí VP, Amin AK, Fisher JB. Radial artery collateral aneurysm: a rare entity resulting from an unusual etiology. Ann Vasc Surg. 1992;6:530-2.

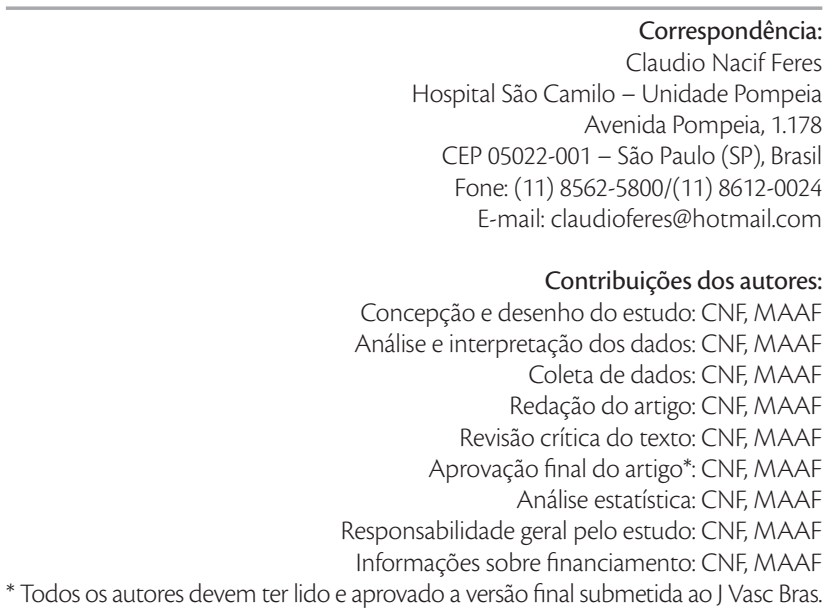

